\title{
Linguistic information aggregation operator and its application in multi-attribute group decision making
}

\author{
Jibin Lan \\ College of Mathematics and Information Science, \\ Guangxi University \\ Nanning, Guangxi, P.R.China \\ lanjibin@gxu.edu.cn \\ Shangfei Lu \\ College of Mathematics and Information Science, \\ Guangxi University \\ Nanning, Guangxi, P.R.China \\ beiyuafei@126.com
}

\author{
Xiaochun Mo \\ College of Mathematics and Information Science, \\ Guangxi University \\ Nanning, Guangxi, P.R.China \\ xiaochunmo211@163.com \\ Zhongxing Wang \\ College of Mathematics and Information Science, \\ Guangxi University \\ Nanning, Guangxi, P.R.China \\ wzx@gxu.edu.cn
}

\begin{abstract}
This paper proposes a new method to deal with the multi-attribute group decision making (MAGDM) problem. Firstly, a new distance measure between two linguistic terms is defined, then a new linguistic information aggregation operator based on the distance measure is proposed and their properties are studied. Secondly, in order to deal with multi-attribute group decision making problem under linguistic environment, a similarity measure between two linguistic preference matrices is proposed. The aggregation operator and the similarity measure are applied to a MAGDM problem. Finally, a numerical example is proposed to prove that the new operator is effective in the MAGDM problems. This method avoid the case that a numerical value times a linguistic information and the results of evaluation are still belongs to the original linguistic term set no matter how to calculate. By using the supporting of the order, the linguistic information can not be lose in the aggregation process.
\end{abstract}

Keywords-Distance measure; linguistic information; aggregation operator; similarity measure; MAGDM

\section{INTRODUCTION}

With the development of modern society, the various types of decision problems become more and more complex in the social economic life. The Multiple Attribute Decision Making (MADM) problem is a type of multi-objective decision problem, which has been found wide applications in economics, management, the military and so on. A very important step for solving MAGDM problem is to aggregate group information. The key to aggregating group information is aggregation functions or aggregation operators, which have been extensively investigated. In real life, due to the complexity of the decision making problems, people prefer to use the linguistic information rather than use the precise value. For example, when people describe the speed of a car, they usually use linguistic variables such as "fast", "normal", "slow" instead of the precise value [1].

For dealing with the linguistic information in the decision making, there have been several methods for aggregate linguistic information: extension principle [2], symbolic method [3], linguistic scale method [4], 2-tuple linguistic representation model [5]. The minimum like tnorms, copulas and quasi-copulas, the maximum like tconorms, dual quasi-copulas, dual copulas, between minimum and maximum like means, weighted means [6], the lexmax (Discrimax) and lexmin (Discrimin) methods [7] and so on. When the aggregation operators are used to deal with the linguistic information, the defined discrete linguistic values will be necessarily extended to the continuous ones as in a simple operation using the subscripts of linguistic terms: $0.5 \otimes l_{2} \oplus 0.5 \otimes l_{3}=l_{2.5}$. In such cases, there is an awareness that $l_{2.5}$ does not have any syntax or semantics assigned, because such a virtual linguistic term makes sense only in comparison and in operation. Nevertheless, the existence of ${ }^{l_{2.5}}$ may affect the effectiveness of linguistic term sets to some extent because linguistic term sets would be the same as uncertain linguistic variables in this situation [8]. The minimum operator and the maximum operator are not satisfied the conditions such as strict-Pareto and restricted compensation. Didier Dubois [7] has presented that a qualitative aggregation operator should meet the three requirements: focus effect, strict-Pareto, restricted compensation. The qualitative aggregation operators can make good use of the characteristic of the linguistic variables, and the computing results are more acceptable.

In this paper, to overcome the above drawbacks, a qualitative aggregation operator is proposed. The structure of the article is showed as follows: In section 2, the linguistic term set will be reviewed and extended, the extended linguistic scale function will be proposed, 
distance measure on the the linguistic term set will be defined. In section 3, a mathematical programming model will be built, a new linguistic information aggregation operator is proposed and its properties will be studied. The operator will be applied to the aggregation processes of the multi-attribute group decision making problems, such as obtaining the opinions of the group and the composite scores of the alternatives. In addition, the aggregation results are still in the original linguistic term set. In section 4 , on the foundation of the proposed distance, a distance and a similarity measure between two matrices are defined. The similarity measure is applied for obtaining the experts' weights. In section 5, The group's opinions matrix is transformed into a 2-tuple matrix, the attribute weights will be computed based on the 2-tuple matrix. In section 6 , an application example will be proposed to prove the new method are effective and acceptable in the MAGDM problems.

\section{PRELIMINARIES}

In this section, the linguistic term set will be reviewed and extended, the extended linguistic scale function will be proposed, distance measure on the the linguistic term set will be defined.

Let $L=\left\{l_{i} \mid i=0,1, \ldots, g\right\},(g=2 k, k \in N)$ be a totally ordered discrete and finite term set. Where $l_{i}$ denotes a possible value for a linguistic variable, $N$ is the set of natural numbers.

Usually, any element of the set $L$ should be satisfied the following four characteristics [9]:

1) Order relation: $l_{i} \leq l_{j}$ if $i \leq j$.

2) Negation operator: $\operatorname{neg}\left(l_{i}\right)=l_{j}$, where $i=g-j$.

3) Max operator: $\max \left\{l_{i} \cdot l_{j}\right\}=l_{i}$ if $i \geq j$.

4) Min operator: $\min \left\{l_{i} \cdot l_{j}\right\}=l_{i}$ if $i \leq j$.

We extend $L$ to $\tilde{L}=\left\{l_{x} \mid x \in[0, g]\right\}$.If $l_{x} \in L$, then we call $l_{x}$ the original term, otherwise, we call $l_{x}$ the virtual term.

Definition 1. Let $\tilde{L}=\left\{l_{x} \mid x \in[0, g]\right\}$ be a extended linguistic term set. For any $l_{x_{1}}, l_{x_{2}} \in \tilde{L}$, a binary relation $\leq$ is defined by

$l_{x_{1}} \leq l_{x_{2}} \Leftrightarrow x_{1} \leq x_{2}$ and $l_{x_{1}}=l_{x_{2}} \Leftrightarrow x_{1}=x_{2}$.

Definition 2. Let $L=\left\{l_{i} \mid i=0,1, \ldots, g\right\}$ be a linguistic term set and $v: L \rightarrow[0,1]$ be a function. For any $l_{i} \in L, v$ is defined by [10]:

$$
v\left(l_{i}\right)=\gamma_{i} .
$$

Where $\gamma_{i} \in[0,1](i=0,1, \ldots, g)$,

$$
0=\gamma_{0}<\gamma_{1}<\ldots<\gamma_{g}=1 \text {. }
$$

$v$ is the linguistic scale function. Clearly, $v$ is a strictly monotonically increasing function, so the inverse function of $v$ exists and is denoted by $v^{-1}$.

We extend the linguistic scale function $v$ to $\tilde{L}$ :

Definition 3. Let $\tilde{L}=\left\{l_{x} \mid x \in[0, g]\right\}$ be a extended linguistic term set and $\tilde{v}: \tilde{L} \rightarrow[0,1]$ be a strictly monotonically increasing continuous function. For any $l_{x_{i}} \in \tilde{L}, \tilde{v}$ is defined by:

$$
\tilde{v}\left(l_{i}\right)=\gamma_{x_{i}},
$$

where $\gamma_{x_{i}} \in[0,1] . \quad$ If $\gamma_{x_{i}} \in L$, then $\tilde{v}\left(l_{i}\right)=v\left(l_{i}\right)$.

$\tilde{v}$ is called the extended linguistic scale function. $v$ is a strictly monotonically increasing continuous function, so the inverse function of $\tilde{v}$ exists and is denoted by $\tilde{v}^{-1}$.

Definition 4. Let $\tilde{L}=\left\{l_{x} \mid x \in[0, g]\right\}$ be a extended linguistic term set, $d: \tilde{L} \times \tilde{L} \rightarrow[0,1]$ be a binary function. For any $l_{x_{1}}, l_{x_{2}} \in \tilde{L}, d$ is defined by

$$
d\left(l_{x_{1}}, l_{x_{2}}\right)=\left|\tilde{v}\left(l_{x_{1}}\right)-\tilde{v}\left(l_{x_{2}}\right)\right| .
$$

Property 1 . Let $\tilde{L}=\left\{l_{x} \mid x \in[0, g]\right\}$ be a extended linguistic term set. For any $l_{x_{1}}, l_{x_{2}}, l_{x_{3}} \in \tilde{L}, d$ satisfies the following five conditions:

1) $0 \leq d\left(l_{x_{1}}, l_{x_{2}}\right) \leq 1$.

2) $d\left(l_{x_{1}}, l_{x_{2}}\right) \leq d\left(l_{x_{1}}, l_{x_{3}}\right), d\left(l_{x_{2}}, l_{x_{3}}\right) \leq d\left(l_{x_{1}}, l_{x_{3}}\right)\left(x_{1} \leq x_{2} \leq x_{3}\right)$.

3) $d\left(l_{x}, l_{x}\right)=0 . \forall l_{x} \in \tilde{L}$.

4) $d\left(l_{x_{1}}, l_{x_{2}}\right)=d\left(l_{x_{2}}, l_{x_{1}}\right)$.

5) $d\left(l_{x_{1}}, l_{x_{2}}\right)+d\left(l_{x_{2}}, l_{x_{3}}\right) \geq d\left(l_{x_{1}}, l_{x_{3}}\right)$.

So $d$ is a distance measure on $\tilde{L}$, and $d$ is also a distance measure on $L$.

\section{A NEW LINGUISTIC INFORMATION AGGREGATION OPERATOR}

Base on the proposed distance measure, a new linguistic information aggregation operator will be defined and its properties will be studied.

Suppose $\left(l_{x_{1}}, l_{x_{2}}, \ldots, l_{x_{n}}\right) \in L^{n}, w=\left(w_{1}, w_{2}, \ldots, w_{n}\right)^{T}$, such that $w_{i}>0, \sum_{i=1}^{n} w_{i}=1$. Consider the function

$$
\begin{gathered}
S\left(\tilde{v}\left(l_{x}\right)\right)=\sum_{i=1}^{n} w_{i}\left(d\left(l_{x_{i}}, l_{x}\right)\right)^{2}=\sum_{i=1}^{n} w_{i}\left(\tilde{v}\left(l_{x_{i}}\right)-\tilde{v}\left(l_{x}\right)\right)^{2} \\
\left.\left.=\mathscr{F}\left(l_{x}\right)-2\left(\sum_{i=1}^{n} w_{i} \mathscr{G} l_{x_{i}}\right)\right) \mathscr{G} l_{x}\right)+\sum_{i=1}^{n} w_{i} \mathscr{G}\left(l_{x_{i}}\right) .
\end{gathered}
$$

Where $x \in[0, g]$.

Obviously, the axis of symmetry about $S\left(\tilde{v}\left(l_{x}\right)\right)$ is $\tilde{v}\left(l_{\bar{x}}\right)=\sum_{i=1}^{n} w_{i} \tilde{v}\left(l_{x_{i}}\right)$, and $\min _{l_{x} \in L} S\left(\tilde{v}\left(l_{x}\right)\right)=S\left(\tilde{v}\left(l_{\bar{x}}\right)\right)$.

Lemma 1. Suppose that $y_{1}, y_{2} \in[0, g], y_{1} \leq y_{2}$, and $\tilde{v}\left(l_{\bar{x}}\right)=\sum_{i=1}^{n} w_{i} \tilde{v}\left(l_{x_{i}}\right) \cdot$

1) If $\tilde{v}\left(l_{y_{2}}\right) \leq \tilde{v}\left(l_{\bar{x}}\right)$, then $S\left(\tilde{v}\left(l_{y_{1}}\right)\right) \geq S\left(\tilde{v}\left(l_{y_{2}}\right)\right)$.

2) If $\tilde{v}\left(l_{y_{1}}^{y_{2}}\right) \geq \tilde{v}\left(l_{\bar{x}}\right)$, then $S\left(\tilde{v}\left(l_{y_{1}}\right)\right) \leq S\left(\tilde{v}\left(l_{y_{2}}\right)\right)$.

3) If $\tilde{v}\left(l_{y_{1}}\right) \leq \tilde{v}\left(l_{\bar{x}}\right) \leq \tilde{v}\left(l_{y_{2}}\right)$, then

If $\tilde{v}\left(l_{\bar{x}}\right)-\tilde{v}\left(l_{y_{1}}\right) \leq \tilde{v}\left(l_{y_{2}}\right)-\tilde{v}\left(l_{\bar{x}}\right)$, then

$S\left(\tilde{v}\left(l_{y_{1}}\right)\right) \leq S\left(\tilde{v}\left(l_{y_{2}}\right)\right)$.

If $\tilde{v}\left(l_{\bar{x}}\right)-\tilde{v}\left(l_{y_{1}}\right) \geq \tilde{v}\left(l_{y_{2}}\right)-\tilde{v}\left(l_{\bar{x}}\right)$, then 


$$
\begin{aligned}
& S\left(\tilde{v}\left(l_{y_{1}}\right)\right) \geq S\left(\tilde{v}\left(l_{y_{2}}\right)\right) . \\
& \text { If } \tilde{v}\left(l_{\bar{x}}\right)-\tilde{v}\left(l_{y_{1}}\right)=\tilde{v}\left(l_{y_{2}}\right)-\tilde{v}\left(l_{\bar{x}}\right) \text {, then } \\
& S\left(\tilde{v}\left(l_{y_{1}}\right)\right)=S\left(\tilde{v}\left(l_{y_{2}}\right)\right) .
\end{aligned}
$$

Proof. Since $S$ is a quadratic function of $\tilde{v}\left(l_{x}\right)$,Lemma 1 hold.

Suppose that $w_{i}>0, \sum_{i=1}^{n} w_{i}=1$, consider the mathematical programming as follows:

$$
\text { (MP) } \min _{l_{x} \in L} f\left(l_{x}\right)=\sum_{i=1}^{n} w_{i}\left(d\left(l_{x_{i}}, l_{x}\right)\right)^{2}
$$

For $\tilde{v}\left(l_{\bar{x}}\right)=\sum_{i=1}^{n} w_{i} \tilde{v}\left(l_{x_{i}}\right)$, since $\tilde{v}$ is a strictly monotonically increasing and continuous function, so its inverse function $v^{-1}$ is also a strictly monotonically increasing and continuous function, denote $l_{\bar{x}}=v^{-1}\left(\sum_{i=1}^{n} w_{i} \tilde{v}\left(l_{x_{i}}\right)\right)$.

Theorem 1. Let $l^{*}$ be the optimal solution of MP.

1)If $l_{\bar{x}} \in L$, then $l^{*}=l_{\bar{x}}$.

2)If $l_{\bar{x}} \notin L$, then there exists a non-negative integer

$k(0 \leq k \leq g-1)$, such that $l_{k}<l_{\bar{x}}<l_{k+1}$.

If $\tilde{v}\left(l_{\bar{x}}\right)-\tilde{v}\left(l_{k}\right)>\tilde{v}\left(l_{k+1}\right)-\tilde{v}\left(l_{\bar{x}}\right)$, then $l^{*}=l_{k+1}$.

If $\tilde{v}\left(l_{\bar{x}}\right)-\tilde{v}\left(l_{k}\right)<\tilde{v}\left(l_{k+1}\right)-\tilde{v}\left(l_{\bar{x}}\right)$, then $l^{*}=l_{k}$.

If $\tilde{v}\left(l_{\bar{x}}\right)-\tilde{v}\left(l_{k}\right)=\tilde{v}\left(l_{k+1}\right)-\tilde{v}\left(l_{\bar{x}}\right)$, then $l^{*}=l_{k}$ or

$l^{*}=l_{k+1}$.

In general, the information provided by experts for each variable must be expressed by a simple linguistic term, the modeling of linguistic information is limited. In order to aggregate limited linguistic information, a new linguistic term aggregation operator is proposed as follows:

Definition 5. Let $\psi_{w}: L^{n} \rightarrow L$ be a multiple mapping.

$\forall\left(l_{x_{1}}, l_{x_{2}}, \ldots, l_{x_{n}}\right) \in L^{n}, \psi_{w}$ is defined by

$$
\psi_{w}\left(l_{x_{1}}, l_{x_{2}}, \ldots, l_{x_{n}}\right)=\max \left\{l_{k} \mid l_{k} \in L, f\left(l_{k}\right)=\min _{l_{x} \in L} f\left(l_{x}\right)\right\} \text {. }
$$

Where $w=\left(w_{1}, w_{2}, \ldots, w_{n}\right)^{T}$, such that $w_{i}>0, \sum_{i=1}^{n} w_{i}=1$.

The aggregation operator $\psi_{w}$ ensures that the results of aggregation belongs to $L$.

Property 2. (Boundedness) For any $\left(l_{x_{1}}, l_{x_{2}}, \ldots, l_{x_{n}}\right) \in L^{n}$, $\min \left(l_{x_{1}}, l_{x_{2}}, \ldots, l_{x_{n}}\right) \leq \psi_{w}\left(l_{x_{1}}, l_{x_{2}}, \ldots, l_{x_{n}}\right) \leq \max \left(l_{x_{1}}, l_{x_{2}}, \ldots, l_{x_{n}}\right)$. Property 3. (Focus effect) Let $\left(l_{x_{1}}, l_{x_{2}}, \ldots, l_{x_{n}}\right)\left(l_{y_{1}}, l_{y_{2}}, \ldots, l_{y_{n}}\right)$ $\in L^{n}$.If $w_{k}$ is sufficiently close to 1 and $l_{x_{k}} \pi l_{y_{k}}$, then $\psi_{w}\left(l_{x_{1}}, l_{x_{2}}, \ldots, l_{x_{n}}\right) \leq \psi_{w}\left(l_{y_{1}}, l_{y_{2}}, \ldots, l_{y_{n}}\right)$.

Property 4. (Monotonicity) Let $\left(l_{x_{1}}, l_{x_{2}}, \ldots, l_{x_{n}}\right)\left(l_{y_{1}}, l_{y_{2}}, \ldots, l_{y_{n}}\right)$

$\in L^{n}$. If $l_{x_{i}} \leq l_{y_{i}}(i=1,2, \ldots, n)$, then

$\psi_{w}\left(l_{x_{1}}, l_{x_{2}}, \ldots, l_{x_{n}}\right) \leq \psi_{w}\left(l_{y_{1}}, l_{y_{2}}, \ldots, l_{y_{n}}\right)$.
Definition 6. Let $L=\left\{l_{i} \mid i=0,1, \ldots, g\right\}$ be a linguistic term set. For any $\left(l_{x_{1}}, l_{x_{2}}, \ldots, l_{x_{n}}\right),\left(l_{y_{1}}, l_{y_{2}}, \ldots, l_{y_{n}}\right) \in L^{n}$, a binary relation $\leq_{\left(w, \widetilde{v}\left(l_{x}\right)\right)}$ on $L^{n}$ is defined by

$$
\begin{gathered}
\left(l_{x_{1}}, l_{x_{2}}, \ldots, l_{x_{n}}\right) \leq_{\left(w, \tilde{v}\left(l_{x}\right)\right)}\left(l_{y_{1}}, l_{y_{2}}, \ldots, l_{y_{n}}\right) \Leftrightarrow \\
\sum_{i=1}^{n} w_{i} \tilde{v}\left(l_{x_{i}}\right) \leq \sum_{i=1}^{n} w_{i} \tilde{v}\left(l_{y_{i}}\right), \text { where } w_{i}>0, \sum_{i=1}^{n} w_{i}=1 .
\end{gathered}
$$

Definition 7. Let $\psi_{w}: L^{n} \rightarrow(L, R)$ be a multiple mapping. For any $\left(l_{x_{1}}, l_{x_{2}}, \ldots, l_{x_{n}}\right) \in L^{n}, \varphi_{w}$ is defined by $\varphi_{w}:\left(l_{x_{1}}, l_{x_{2}}, \ldots, l_{x_{n}}\right) \rightarrow\left(\psi_{w}\left(l_{x_{1}}, l_{x_{2}}, \ldots, l_{x_{n}}\right), \sum_{i=1}^{n} w_{i} \tilde{v}\left(l_{x_{i}}\right)\right)$.

Where $w_{i}>0, \sum_{i=1}^{n} w_{i}=1$.

2-tuple $\left(\psi_{w}\left(l_{x_{1}}, l_{x_{2}}, \ldots, l_{x_{n}}\right), \sum_{i=1}^{n} w_{i} \widetilde{v}\left(l_{x_{i}}\right)\right)$ represents the aggregating result of the linguistic information $\left(l_{x_{1}}, l_{x_{2}}, \ldots, l_{x_{n}}\right) \in L^{n} \cdot \psi_{w}\left(l_{x_{1}}, l_{x_{2}}, \ldots, l_{x_{n}}\right)$ represents the aggregating result of linguistic term, $\sum_{i=1}^{n} w_{i} \tilde{v}\left(l_{x_{i}}\right)$ is a numerical value supporting of the order relation $\leq_{\left(w, \tilde{v}\left(l_{x}\right)\right)}$ on $L^{n}$.

\section{A SIMILARITY MEASURE BETWEEN TWO PREFERENCE MATRICES}

In this section, a similarity measure between two linguistic terms will be used to obtain the experts' weights, then the group's opinions are received.

Let $A=\left\{A_{1}, A_{2}, \ldots, A_{m}\right\}$ be a discrete set of alternatives, $C=\left\{C_{1}, C_{2}, \ldots, C_{n}\right\}$ be the set of attributes, $E=\left\{E_{1}, E_{2}, \ldots, E_{r}\right\}$ be the set of experts. Suppose that $Q=\left\{Q_{1}, Q_{2}, \ldots, Q_{r}\right\}$ is the set of preference matrices given by the decision makers. $Q^{p}=\left(l_{i j}^{p}\right)_{m \times n},(p=1,2, \ldots, r)$,

Where $l_{i j}^{p} \in L$ is a linguistic preference value for alternatives $A_{i}(i=1,2, \ldots, m)$ with respect to the attributes $C_{j}(j=1,2, \ldots, n)$ given by the experts $E^{p}(p=1,2, \ldots, r)$. Definition 8 . Let $D: Q \times Q \rightarrow R$ be a binary function. For any $Q^{p}, Q^{q} \in Q, D$ is defined by

$$
D\left(Q^{p}, Q^{q}\right)=\frac{1}{m n} \sum_{i=1}^{m} \sum_{j=1}^{n} d\left(l_{i j}^{p}, l_{i j}^{q}\right) \cdot
$$

Definition 9. Let $S: Q \times Q \rightarrow[0,1]$ be a binary function.

For any $Q^{p}, Q^{q} \in Q, S$ is defined by

$$
S\left(Q^{p}, Q^{q}\right)=1-D\left(Q^{p}, Q^{q}\right) .
$$

Where $D\left(Q^{p}, Q^{q}\right)$ is the distance measure between $Q^{p}$ and $Q^{q} \cdot S\left(Q^{p}, Q^{q}\right)$ is the similarity degree between $Q^{p}$ and $Q^{q}$.

Obviously, the bigger of $D\left(Q^{p}, Q^{q}\right)$ is, the smaller of $S\left(Q^{p}, Q^{q}\right)$ is. Especially if $Q^{p}=Q^{q}$, then 
$D\left(Q^{p}, Q^{q}\right)=0 \Rightarrow S\left(Q^{p}, Q^{q}\right)=1$.

The total similarity degree between decision maker $E^{p}(p=1,2, \ldots, r)$ and the other decision makers is:

$$
S^{p}=\sum_{q=1}^{r} S\left(Q^{p}, Q^{q}\right), p \neq q .
$$

The total degree of similarity among experts $E^{p}$ $(p=1,2, \ldots, r)$ is:

$$
\bar{S}=\sum_{p=1}^{r} S^{p}
$$

Since the higher level of similarity degree, the higher weight is given to the expert, so the weight of expert $E^{p}$ can be denoted by:

$$
w_{E^{p}}=S^{p} / \bar{S} .
$$

\section{APPLICATION IN THE MAGDM}

In this section, the deviation among the alternatives will be defined and attribute weights will be obtained.

In the multiple attribute decision making, these alternatives are compared by the synthesized attribute values. The distance is used as the deviation. If all the alternatives under the value of the attribute with the smaller deviation, it means that the attribute has less affection in the alternatives' sorting. On the contrary, if the attribute has a larger deviation in the value of the attribute for all the alternatives, it means that the attribute will play an important role in the sorting. Therefore, how to order the alternatives will be considered, the value of the attribute which has a larger distance should be given a larger weight [11].

In the MAGDM problems, by using formulas (9-13) and operator $w_{\psi}$, the the group's opinions are received. As the process of aggregating group's opinions may lose a lot of linguistic information. In order to avoid this drawback, base on Definition 7, we transform $l^{G}(i, j)$ into 2-tuple $\left(l^{G}(i, j), \sum_{p=1}^{r} w_{E^{p}} \tilde{v}\left(l_{x_{i j}}^{p}\right)\right) \cdot$

For attributes $C_{j}(j=1,2, \ldots, n), V_{j}$ represents the deviation among the alternatives:

$$
V_{j}=\sum_{i=1}^{m} \sum_{t=1}^{m}\left|\sum_{p=1}^{r} w_{E^{p}} \tilde{v}\left(l_{x_{i j}}^{p}\right)-\sum_{p=1}^{r} w_{E^{p}} \tilde{v}\left(l_{x_{i j}}^{p}\right)\right| .
$$

Since a larger deviation should be given a larger weight, the weights of attributes $C_{j}$ can be expressed by:.

$$
w_{j}^{\prime}=V_{j} / \sum_{j=1}^{n} V_{j} .
$$

Base on the numerical value supporting of the order relation, rank the alternatives by the value of $\sum_{j=1}^{n} w_{j}^{\prime}\left(\sum_{p=1}^{r} w_{E^{p}} \tilde{v}\left(l_{x_{i j}}^{p}\right)\right)$.

The steps for solving the MAGDM problems are expressed as follows:

Step 1. Chose a linguistic scale $\tilde{v}\left(l_{x}\right)$.

Step 2.The experts' weights are computed by the formulas (9-13).
Step 3. Utilize model MP to obtain the group's opinions matrix $Q^{G}=\left(l^{G}(i, j)\right)$, transform $l_{i j}^{G}$ into 2-tuple

$\left(l^{G}(i, j), \sum_{p=1}^{r} w_{E^{p}} \tilde{v}\left(l_{x_{i j}}^{p}\right)\right) \cdot$

Step 4. The deviation $V_{j}$ among the alternatives are calculated by formula (14).

Step 5. Use equation (15) to compute the each attribute weight..

Step 6. Rank the alternatives by the value

of $\sum_{j=1}^{n} w_{j}^{\prime}\left(\sum_{p=1}^{r} w_{E^{p}} \tilde{v}\left(l_{x_{i j}}^{p}\right)\right)$.

\section{NUMERICAL EXAMPLE}

Let us suppose there is a talent search show, which wants to select the best talented person. There are three experts $E^{p}(p=1,2,3)$ to evaluate the five competitors $A_{i}(i=1,2,3,4,5)$, according to the following four criterion: $C_{1}$ is introduce myself; $C_{2}$ is self-cultivation; $C_{3}$ is stage performance; $C_{4}$ is competence of meeting an emergency. The five competitors $A_{i}(i=1,2,3,4,5)$ are to be evaluated using the linguistic term set:

$L=\left\{l_{0}=\right.$ very poor, $l_{1}=$ poor,$l_{2}=$ slightly poor, $l_{3}=$ fair, $l_{4}=$ slightly good, $l_{5}=$ good, $l_{6}=$ very good $\}$.

The preference matrices given by the three experts are $Q^{1}, Q^{2}, Q^{3}$ showed as follows:

$$
\begin{array}{llllllll}
C_{1} & C_{2} & C_{3} & C_{4} & C_{1} & C_{2} & C_{3} & C_{4}
\end{array}
$$

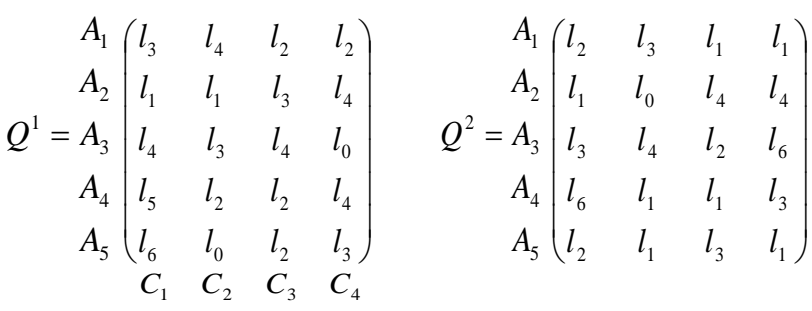

$$
Q^{3}=\begin{array}{r}
A_{1} \\
A_{2} \\
A_{3} \\
A_{4} \\
A_{5}
\end{array}\left(\begin{array}{llll}
l_{4} & l_{2} & l_{1} & l_{5} \\
l_{1} & l_{4} & l_{2} & l_{4} \\
l_{5} & l_{1} & l_{4} & l_{2} \\
l_{3} & l_{5} & l_{6} & l_{1} \\
l_{3} & l_{3} & l_{4}
\end{array}\right)
$$

Step 1. Take the linguistic scale $\tilde{v}\left(l_{x}\right)=x / 6$.

Step 2. By using formulas (9), distance measures between two linguistic preference matrices are calculated:

$$
\begin{aligned}
& D\left(Q^{1}, Q^{2}\right)=0.2333, \quad D\left(Q^{1}, Q^{3}\right)=0.2750, \\
& D\left(Q^{2}, Q^{3}\right)=0.3417 .
\end{aligned}
$$

By using formulas (10), similarity measures between two linguistic preference matrices are:

$$
\begin{aligned}
& S\left(Q^{1}, Q^{2}\right)=1-D\left(Q^{k}, Q^{1}\right)=0.7667, \\
& S\left(Q^{1}, Q^{3}\right)=1-D\left(Q^{k}, Q^{2}\right)=0.7250, \\
& S\left(Q^{2}, Q^{3}\right)=1-D\left(Q^{k}, Q^{3}\right)=0.6583 .
\end{aligned}
$$

By using formulas (11), the total similarity degree between decision maker $E^{p}(p=1,2,3)$ and the other decision makers are: 


$$
\begin{aligned}
& S^{1}=S\left(Q^{1}, Q^{2}\right)+S\left(Q^{1}, Q^{3}\right)=1.4917, \\
& S^{2}=S\left(Q^{1}, Q^{2}\right)+S\left(Q^{2}, Q^{3}\right)=1.4250, \\
& S^{3}=S\left(Q^{1}, Q^{3}\right)+S\left(Q^{2}, Q^{3}\right)=1.3833 .
\end{aligned}
$$

Base on formulas (12), the total degree of similarity among experts $E^{p}(p=1,2,3)$ is: $\quad \bar{S}=\sum_{p=1}^{3} S^{p}=4.3000$.

Base on formulas (13), the experts' weights are:

$$
w^{1}=0.3469, w^{2}=0.3314, w^{3}=0.3217 \text {. }
$$

Step 3. By using the MP model, the group's opinions are obtained:

$$
\begin{aligned}
& \begin{array}{llll}
C_{1} & C_{2} & C_{3} & C_{4}
\end{array} \\
& Q^{G}=\begin{array}{r}
A_{2} \\
A_{3} \\
A_{4}
\end{array}\left(\begin{array}{llll}
l_{3} & l_{3} & l_{1} & l_{3} \\
l_{1} & l_{2} & l_{3} & l_{4} \\
A_{5} & l_{3} & l_{3} & l_{3} \\
l_{5} & l_{3} & l_{3} & l_{3} \\
l_{4} & l_{1} & l_{3} & l_{3}
\end{array}\right)
\end{aligned}
$$

the aggregation results of group's opinions $l^{G}(i, j)$ can be transform into 2-tuple $\left(l^{G}(i, j), \sum_{p=1}^{r} w_{E^{p}} \widetilde{v}\left(l_{x_{i j}}^{p}\right)\right)$ :

$C_{1}$

$$
\begin{aligned}
& A_{1}\left(\left(l_{3}, 0.4984\right) \quad\left(l_{3}, 0.5042\right) \quad\left(l_{1}, 0.2245\right) \quad\left(l_{3}, 0.4390\right)\right. \\
& A_{2}\left(\begin{array}{lllll}
\left(l_{1}, 0.1667\right) & \left(l_{2}, 0.2723\right) & \left(l_{3}, 0.5016\right) & \left(l_{4}, 0.4390\right)
\end{array}\right. \\
& \begin{array}{lllll}
A_{3} & \left(l_{4}, 0.6651\right) & \left(l_{3}, 0.4480\right) & \left(l_{3}, 0.5562\right) & \left(l_{3}, 0.4386\right)
\end{array} \\
& A_{4} \quad\left(l_{5}, 0.8350\right) \quad\left(l_{3}, 0.4390\right) \quad\left(l_{3}, 0.4926\right) \quad\left(l_{3}, 0.4506\right) \\
& A_{5}\left(\begin{array}{llll}
\left(l_{4}, 0.6182\right) & \left(l_{1}, 0.1089\right) & \left(l_{3}, 0.4422\right) & \left(l_{3}, 0.4432\right)
\end{array}\right.
\end{aligned}
$$

Step 4. Base on formula (14), calculate the deviation $V_{j}(1,2,3,4)$ among the alternatives:

$$
V_{1}=6.0132, V_{2}=3.8652, V_{3}=2.8912, V_{4}=1.8712 \text {. }
$$

Step 5. Use equation (15) to compute the each attribute weights:

$$
w^{\prime}=(0.4107,0.2640,0.1975,0.1278)^{T} .
$$

Step 6. Rank the alternatives by the value of

$$
\begin{aligned}
& \left.\sum_{j=1}^{n} w_{j}^{\prime}\left(\sum_{p=1}^{r} w_{E^{p}} 9 k d_{x_{1 j}}^{p}\right)\right)=0.4382 \text {, } \\
& \left.\sum_{j=1}^{n} w_{j}^{\prime}\left(\sum_{p=1}^{r} w_{E^{p}} 19 d d_{x_{2 j}}^{p}\right)\right)=0.3246 \text {, } \\
& \sum_{j=1}^{n} w_{j}^{\prime}\left(\sum_{p=1}^{r} w_{E^{p}} 9\left(d_{x_{3 j}}^{p}\right)\right)=0.5573 \text {, } \\
& \sum_{j=1}^{n} w_{j}^{\prime}\left(\sum_{p=1}^{r} w_{E^{p}} \mathcal{M}\left(l_{x_{4 j}}^{p}\right)\right)=0.6137 \text {, } \\
& \sum_{j=1}^{n} w_{j}^{\prime}\left(\sum_{p=1}^{r} w_{E^{p}} \mathscr{g}\left(d_{x_{5_{j}}}^{p}\right)\right)=0.4266 \text {. }
\end{aligned}
$$

Since $0.6137>0.5573>0.4382>0.4266>0.3246$, then

$$
A_{4} \phi A_{3} \phi A_{1} \phi A_{5} \phi A_{2}
$$

So the best alternative is $A_{4}$.

By using the 2-tuple linguistic representation model [5] to deal with the problem above, the composite scores of $A_{1}, A_{2}, A_{3}, A_{4}, A_{5}$ are $\left(l_{3},-0.37\right),\left(l_{2},-0.05\right),\left(l_{3}, 0.34\right)$, $\left(l_{4},-0.34\right),\left(l_{2},-0.44\right)$ respectively, then

$$
A_{4} \phi A_{3} \phi A_{1} \phi A_{5} \phi A_{2} \text {. }
$$

The results of the computing are the same, however, in the process of aggregating linguistic information, there exist some problems in the symbolic method, such as $0.5 \otimes l_{2} \oplus 0.5 \otimes l_{3}=l_{2.5}$ do not make sense because $l_{2.5} \notin L$.

\section{CONCLUSIONS}

This paper has proposed a new operator and its properties has been studied. Furthermore, the new operator is applied to obtain the group's opinions in the MAGDM problems. The linguistic information still belongs to the original linguistic term set no matter how to calculate. In order to avoid losing linguistic information in the process of aggregating group's opinions. The group's opinions matrix is transformed into a 2-tuple matrix. According to the numerical value supporting of the order relation, the best alternative is obtained. In the future, we will find other distances which can deal with the linguistic scale function, then obtain the new operators with the better properties.

\section{ACKNOWLEDGMENT}

This research was supported by National Science Foundation of China (No. 71261001) and Youth Science Foundation Project of China (No. 71201037).

\section{REFERENCES}

[1] Umano M, Hatono I, Tamura H, Linguistic labels for expressing fuzzy preference relations in fuzzy group decision making, Systems, Man, and Cybernetics, Part B: Cybernetics, IEEE Transactions on, 1998,28(2): 205-218.

[2] Degani R, Bortoaln G., The problem of linguistic approximation in clinical decision making, Approximate Reasoning, 1988, 2: 143 162.

[3] Herrera F, Herrera-Viedma E, Martinez L., Direct approach processes in group decision making using linguistic OWA operators. Fuzzy sets and Systems, 1996, 79(2): 175-190.

[4] Xu, Z S, Group decision making based on multiple types of linguistic preference relations, Information Sciences, 2008, 178: 452-467.

[5] Dong Y Ch, Xu Y F, Li H Y, Feng B., The OWA-based consensus operator under linguistic representation models using position indexes, European Journal of Operational Research, 2010, 203: 455-463.

[6] Jian-qiang Wang, Jing Wang, et al, An outranking approach for multi-criteria decision-making with hesitant fuzzy linguistic term sets, Information Sciences 280 (2014) 338-351.

[7] Didier Dubois, The role of fuzzy sets in decision sciences: Old techniques and new directions. Fuzzy Sets and Systems 184 (2011) 3-28.

[8] G.Beliakov, A.Pradera, T.Calvo, Aggregation Functions: A Guide for Practitioners, Springer, Studies in Fuzziness and Soft Computing, Berlin, Heidelberg, 2007.

[9] F. Herrera, L. Martinez, P.J. Sachez, Managing non-homogeneous information in group decision-making, European Journal of Operational Research, 166 (1) (2005) 115-132.

[10] Jian-qiang Wang, Jing Wang, et al, Interval-valued hesitant fuzzy linguistic sets and their applications in multi-criteria decisionmaking problems, Information Sciences 288 (2014) 55-72

[11] Herrera, F., Herrera-Viedma, E., Linguistic decision analysis: Steps for solving decision problems under linguistic information. Fuzzy Sets and Systems, 115, (2000) 67-82. 